International Journal of Pure and Applied Mathematics

Volume 96 No. 2 2014, 229-234

ISSN: 1311-8080 (printed version); ISSN: 1314-3395 (on-line version)

url: http://www.ijpam.eu

doi: http://dx.doi.org/10.12732/ijpam.v96i2.6

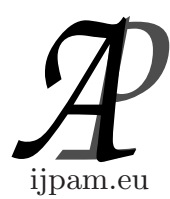

\title{
ANALYTIC SOLUTION OF A NONLINEAR BLACK-SCHOLES EQUATION WITH PRICE SLIPPAGE
}

\author{
Joseph Eyang'an Esekon \\ Department of Statistics and Actuarial Science \\ Maseno University \\ P.O. Box 333-40105, Maseno, KENYA
}

\begin{abstract}
We study a nonlinear Black-Scholes partial differential equation whose nonlinearity is as a result of transaction cost and a price slippage impact that lead to market illiquidity with feedback effects. After reducing the equation into a second-order nonlinear partial differential equation, we find that the assumption of a traveling wave profile to the second-order equation reduces it further to ordinary differential equations. Solutions to all these transformed equations facilitate an analytic solution to the nonlinear Black-Scholes equation. We finally show that the option is always more volatile compared to the stock when $\frac{1 \mp \sqrt{1-(1-\alpha)^{2}}}{(1-\alpha)^{2}}<\frac{S_{0}}{S} e^{r t}$.
\end{abstract}

AMS Subject Classification: 35A09, 35A20, 62P05

Key Words: analytic solution, feedback effects, illiquid markets, transaction cost, price slippage

\section{Introduction}

Two primary assumptions are used in formulating classical arbitrage pricing theory: frictionless and competitive markets. There are no transaction costs and restrictions on trade in a frictionless market. In a competitive market, a trader can buy or sell any quantity of a security without changing its price.

Received: April 15, 2014

(c) 2014 Academic Publications, Ltd. url: www.acadpubl.eu 
Restrictions on trade are imposed when we have extreme market conditions. In particular, purchases/short sales are not permitted when the market has a surplus/shortage.

The notion of liquidity risk is introduced on relaxing the assumptions above.

The purpose of this paper is to obtain an analytic solution of the nonlinear Black-Scholes equation arising from the combination of transaction cost and a price slippage impact by Bakstein and Howison in [1]. This is done by substitutions and transformations, which give a second-order nonlinear partial differential equation. Assuming a traveling wave solution to the second-order equation reduces it further to ordinary differential equations (ODEs). All these transformed equations are solved to obtain an analytic solution to the nonlinear Black-Scholes equation.

This paper is organized as follows. Section 2 describes the nonlinear BlackScholes PDE used for modelling illiquid markets with a price slippage impact. The smooth solution to the nonlinear Black-Scholes equation is presented in Section 3. Section 4 concludes the paper.

\section{Bakstein and Howison (2003) Equation}

In this section, we will consider the continuous-time feedback effects equation for illiquid markets by Bakstein and Howison in [1]. Two assets are used in the model: a bond (or a risk-free money market account with spot rate of interest $r \geq 0$ ) whose value at time $t$ is $B_{t} \equiv 1$, and a stock. The stock is assumed to be risky and illiquid while the bond is assumed to be riskless and liquid. This equation (see Theorem 3.1 of [1]) is given by

$$
u_{t}+\frac{1}{2} \sigma^{2} S^{2} u_{S S}\left(1+2 \rho S u_{S S}\right)+\frac{1}{2} \rho^{2}(1-\alpha)^{2} \sigma^{2} S^{4} u_{S S}^{3}+r S u_{S}-r u=0,
$$

where $S$ is the price of the stock, $\rho \geq 0$ is a measure of the liquidity of the market, $\sigma$ is volatility, $u(S, t)$ is the option price and $\alpha$ is a measure of the price slippage impact of a trade felt by all participants of a market (see Theorem 3.1 of $[1])$.

For instance the terminal condition for a European call option is given by

$$
h\left(S_{T}\right)=u(S, T)=\max \{S-K, 0\} \quad \text { for } \quad S \geq 0,
$$

where $K>0$ is the striking price and $h\left(S_{T}\right)$ is a terminal claim whose hedge cost, $u\left(S_{t}, t\right)$, is the solution to $(2.1)$. The boundary conditions for the option are as follows:

$$
u(0, t)=0 \quad \text { for } \quad 0 \leq t \leq T
$$




$$
u(S, t) \sim S-K e^{-r(T-t)} \quad \text { as } \quad S \rightarrow \infty .
$$

We take the last condition to mean that

$$
\lim _{S \rightarrow \infty} \frac{u(S, t)}{S-K e^{-r(T-t)}}=1
$$

uniformly for $0 \leq t \leq T$ with the constraint $u(S, t) \geq 0$.

Liquidity in (2.1) has been defined through a combination of transaction cost and a price slippage impact. Due to $\rho$, bid-ask spreads dominate the price elasticity effect. When $\alpha=1$, this corresponds to no slippage and equation (2.1) reduces to the $\mathrm{PDE}$ given by

$$
u_{t}+\frac{1}{2} \sigma^{2} S^{2} u_{S S}\left(1+2 \rho S u_{S S}\right)+r S u_{S}-r u=0 .
$$

The solution to equation (2.2) is found in Theorem 3.0.2 of [3] for $r>0$, and in Theorem 3.2 of [4] and Theorem 4.1 of [5] for $r=0$.

The magnitude of the market impact is determined by $\rho S$. Large $\rho$ implies a big market impact of hedging. If $\rho=0$, the asset's price in equation (2.1) follows the standard Black-Scholes model in [2] with constant volatility $\sigma$.

\section{Smooth Solution to the Bakstein and Howison (2003) Equation}

Lemma 3.1. If $\nu(\xi)$ is a twice continuously differentiable function, and $x$ and $t$ are the spatial and time variables respectively, then there exists a traveling wave solution to the equation,

$$
V_{t}+\frac{1}{2} \sigma^{2}\left(V_{x x}+V_{x}\right)\left(1+2\left(V_{x x}+V_{x}\right)\right)+\frac{1}{2}(1-\alpha)^{2} \sigma^{2}\left(V_{x x}+V_{x}\right)^{3}+r V_{x}=0
$$

in $\mathbb{R} \times(0, \infty)$ of the form

$$
V(x, t)=\nu(\xi) \quad \text { where } \quad \xi=x-c t, \quad \xi \in \mathbb{R}
$$

for $0 \leq \alpha<1, \quad 1<\alpha \leq 2, \quad r, \sigma, t>0 \quad$ and $\quad x \in \mathbb{R}$ such that $V(x, t)$ is a traveling wave of permanent form which translates to the right with constant speed $c>0$.

Proof. Applying the chain rule to (3.2) gives

$$
V_{t}=-c \nu^{\prime}(\xi), \quad V_{x}=\nu^{\prime}(\xi), \quad \text { and } \quad V_{x x}=\nu^{\prime \prime}(\xi),
$$

where the prime denotes $\frac{d}{d \xi}$. Substituting these expressions into (3.1), we conclude that $\nu(\xi)$ must satisfy the nonlinear second order ODE

$$
-c \nu^{\prime}+\frac{1}{2} \sigma^{2}\left(\nu^{\prime \prime}+\nu^{\prime}\right)\left(1+2\left(\nu^{\prime \prime}+\nu^{\prime}\right)\right)+\frac{1}{2}(1-\alpha)^{2} \sigma^{2}\left(\nu^{\prime \prime}+\nu^{\prime}\right)^{3}+r \nu^{\prime}=0
$$


in $\mathbb{R}$ and hence $V(x, t)$ solves $(3.1)$.

By setting $c=r$, the equation resulting from (3.3) can now be solved in a closed-form by first writing it as

$$
(1-\alpha)^{2}\left(\nu^{\prime \prime}+\nu^{\prime}\right)^{2}+2\left(\nu^{\prime \prime}+\nu^{\prime}\right)+1=0 \quad \text { in } \quad \mathbb{R},
$$

where $(1-\alpha)^{2} \neq 0$.

The quadratic equation above is solved to get

$$
\nu^{\prime \prime}+\nu^{\prime}=\frac{-1 \pm \sqrt{1-(1-\alpha)^{2}}}{(1-\alpha)^{2}}, \quad 0 \leq \alpha<1, \quad 1<\alpha \leq 2
$$

Upon integration we get the variable separable standard form (see [6])

$$
\nu^{\prime}=e^{\xi_{0}-\xi}+\frac{-1 \pm \sqrt{1-(1-\alpha)^{2}}}{(1-\alpha)^{2}}, \quad 0 \leq \alpha<1, \quad 1<\alpha \leq 2, \quad \xi_{0}, \xi \in \mathbb{R}
$$

where $\xi_{0}$ is a constant of integration. This is the first order linear autonomous and separable ODE whose solution upon integration is given by

$$
\nu(\xi)=-e^{\xi_{0}-\xi}+\frac{-1 \pm \sqrt{1-(1-\alpha)^{2}}}{(1-\alpha)^{2}} \xi+\delta
$$

for $0 \leq \alpha<1,1<\alpha \leq 2, \xi_{0}, \xi \in \mathbb{R}$, where $\delta$ is another constant of integration. Applying the initial condition

$$
\nu(0)=0
$$

to the equation above gives

$$
\delta=e^{\xi_{0}}
$$

Hence

$$
\nu(\xi)=-e^{\xi_{0}-\xi}+\frac{-1 \pm \sqrt{1-(1-\alpha)^{2}}}{(1-\alpha)^{2}} \xi+e^{\xi_{0}}
$$

for $0 \leq \alpha<1,1<\alpha \leq 2, \xi_{0}, \xi \in \mathbb{R}$. Substitution gives

$$
V(x, t)=-e^{x_{0}-(x-r t)}+\frac{-1 \pm \sqrt{1-(1-\alpha)^{2}}}{(1-\alpha)^{2}}(x-r t)+e^{x_{0}},
$$

for $0 \leq \alpha<1,1<\alpha \leq 2, x_{0}, x \in \mathbb{R}, r>0, t \geq 0$ since $\xi_{0}=x_{0}-c \cdot 0=x_{0}$ and $c=r$.

Theorem 3.2. If $V(x, t)$ is any positive solution to the nonlinear equation

$$
V_{t}+\frac{1}{2} \sigma^{2}\left(V_{x x}+V_{x}\right)\left(1+2\left(V_{x x}+V_{x}\right)\right)+\frac{1}{2}(1-\alpha)^{2} \sigma^{2}\left(V_{x x}+V_{x}\right)^{3}+r V_{x}=0
$$


in $\mathbb{R} \times[0, \infty)$ then

$$
u(S, t)=\frac{1}{\rho}\left[\left(\frac{-1 \pm \sqrt{1-(1-\alpha)^{2}}}{(1-\alpha)^{2}}\left\{\ln \left(\frac{S}{K}\right)-r t\right\}+\frac{S_{0}}{K}\right) S-S_{0} e^{r t}\right]
$$

in $\mathbb{R} \times[0, \infty)$ solves the nonlinear Black-Scholes equation

$$
u_{t}+\frac{1}{2} \sigma^{2} S^{2} u_{S S}\left(1+2 \rho S u_{S S}\right)+\frac{1}{2} \rho^{2}(1-\alpha)^{2} \sigma^{2} S^{4} u_{S S}^{3}+r S u_{S}-r u=0
$$

for $r, K, S, S_{0}, \sigma, \rho>0, t \geq 0,0 \leq \alpha<1$, and $1<\alpha \leq 2$, where $S_{0}$ is the initial stock price.

Proof. To obtain the solution to equation (3.6) we apply the transformations $x=\ln \left(\frac{S}{K}\right)$ and $V(x, t)=\rho \frac{u(S, t)}{K e^{x}}$ to get

$$
u_{t}=\frac{S}{\rho} V_{t}, \quad u_{S}=\frac{1}{\rho}\left(V_{x}+V\right), \quad u_{S S}=\frac{1}{\rho S}\left(V_{x x}+V_{x}\right) .
$$

Substituting these expressions into (3.6) gives (3.1). Hence, applying the transformations above into (3.4) gives (3.5).

Remark 3.3. From equation (3.5), it is clear that when $\frac{1 \mp \sqrt{1-(1-\alpha)^{2}}}{(1-\alpha)^{2}}<$ $\frac{S_{0}}{S} e^{r t}$ then $\frac{S u_{S}}{u}$ is always greater than one as in $[2,3,4]$. This shows that the option is always more volatile than the stock when this inequality holds.

\section{Conclusion}

We have studied the hedging of derivatives in the presence of transaction cost and a price slippage impact that lead to market illiquidity with feedback effects. Assuming the solution of a forward wave, a classical solution for the nonlinear Black-Scholes equation was found. The solution obtained can be used for pricing a European call option at time $t \geq 0$. It is clear that when $\frac{1 \mp \sqrt{1-(1-\alpha)^{2}}}{(1-\alpha)^{2}}<\frac{S_{0}}{S} e^{r t}$ this solution supports the comments in $[2,3,4]$ that the option is always more volatile than the stock.

In conclusion, further research needs to be done to find out the solution to the equation when $c \neq r$. Another part of our interest is the use of the put-call-parity to obtain the solution of a put option. 


\section{References}

[1] D. Bakstein, S. Howison, A non-arbitrage liquidity model with observable parameters for derivatives, Working paper, (2003).

[2] F. Black, M. Scholes, The pricing of options and corporate liabilities, The Journal of Political Economy, 81, No. 3 (1973), 637-654.

[3] J. E. Esekon, Analytic solution of a nonlinear Black-Scholes equation, International Journal of Pure and Applied Mathematics, 82, No. 4 (2013), $547-555$.

[4] J. E. Esekon, A particular solution of a nonlinear Black-Scholes partial differential equation, International Journal of Pure and Applied Mathematics, 81, No. 5 (2012), 715-721.

[5] J. Esekon, S. Onyango, N.O. Ongati, Analytic solution of a nonlinear Black-Scholes partial differential equation, International Journal of Pure and Applied Mathematics, 61, No. 2 (2010), 219-230.

[6] J. D. Logan, An Introduction to Nonlinear Partial Differential Equations, Second Edition, John Wiley and Sons, Inc., Hoboken, New Jersey, USA (2008). 